\title{
A SEGUNDA LEI DA TERMODINÂMICA
}

Martina Costa Reis e Adalberto Bono Maurizio Sacchi Bassi*

Departamento de Físico-Química, Instituto de Química, Universidade Estadual de Campinas, CP 6154, 13083-970 Campinas SP, Brasil

Recebido em 26/6/11; aceito em 11/11/11; publicado na web em 13/1/12

\begin{abstract}
THE SECOND LAW OF THERMODYNAMICS. Considering intrinsic characteristics of the system exclusively, both statistical and information theory interpretations of the second law are used to provide more comprehensive meanings for the concepts of entropy, temperature, and Helmholtz and Gibbs energies. The coherence of Clausius inequality to these concepts is emphasized. The aim of this work is to re-discuss the second law of thermodynamics in accordance to homogeneous processes thermodynamics, a temporal science which is the very special oversimplification of continuum mechanics for spatially constant intensive properties.
\end{abstract}

Keywords: entropy; temperature; Clausius inequality.

\section{INTRODUÇÃO}

A origem da segunda lei da termodinâmica é associada ao trabalho do físico Sadi Carnot (1796-1832), o qual estava particularmente interessado na eficiência de máquinas térmicas operando sob condições ideais. Em seu livro Refléxions sur la Puissance Motrice du Feu et sur les Machines Propres à Développer Cette Puissance, ${ }^{1}$ Carnot demonstra que, para produzir trabalho em um processo cíclico, não é suficiente apenas absorção de calor pela máquina térmica, porque também é necessário que a máquina emita alguma quantidade de calor.

Anos após a divulgação do trabalho de Carnot, William Thomson (1824-1870), também conhecido como Lord Kelvin, afirmou que, se a máquina térmica trocar calor com suas vizinhanças em um processo cíclico e tais vizinhanças se encontrarem em uma única temperatura, então a máquina não poderá produzir trabalho. Em 1850, outra formulação para a segunda lei da termodinâmica foi proposta por Rudolph Clausius (1822-1888), o qual percebeu que o principal axioma de Carnot envolve, para processos cíclicos, a conversibilidade de calor em trabalho e vice-versa, demonstrada poucos anos antes, por meio de uma série de experimentos, por James Joule (1818-1889). Clausius estabeleceu que a temperatura da fonte que absorve o calor emitido pela máquina que produz trabalho é menor do que a temperatura da fonte que cede calor à máquina. ${ }^{2}$

Posteriormente, em 1862, Clausius propôs a existência de alguma quantidade física, atualmente denominada entropia, cuja variação se encontre relacionada ao sentido em que os processos ocorrem. Além disso, ao garantir que a passagem de calor de um corpo frio para um corpo quente não pode ocorrer espontaneamente, reafirmou o enunciado da segunda lei. ${ }^{3}$ Com base neste enunciado, Serrin $^{4}$ demonstrou que, para um processo cíclico, calor absorvido e emitido, respectivamente nas temperaturas $\mathrm{T}_{1}$ e $\mathrm{T}_{2}$, sendo $\mathrm{T}_{1}<\mathrm{T}_{2}$, implica em trabalho realizado sobre o sistema. Durante muito tempo, a formulação matemática da segunda lei da termodinâmica, em especial o real significado da quantidade entropia, permaneceram incompreendidos. ${ }^{5,6}$

Em 1872 Ludwig Boltzmann (1844-1906) deduziu, a partir da teoria cinética dos gases proposta por James Maxwell (1831-1879), o seu famoso teorema $\mathrm{H}$, que pode ser entendido como um análogo da segunda lei. De acordo com Boltzmann, a definição do estado de um gás perfeito, a cada instante, envolve a velocidade e a posição de cada molécula constituinte naquele instante, podendo um mesmo estado

*e-mail: bassi@iqm.unicamp.br corresponder a várias configurações microscópicas, ou microestados. No teorema $\mathrm{H}$, Boltzmann considerou que, quanto maior o número de microestados correspondentes a um estado, maior a probabilidade de este estado ocorrer, o que conduz ao conceito de processo irreversível e, para sistema isolado, também ao de entropia. A segunda lei ganhou, assim, a possibilidade de ser interpretada como uma tendência estatística que, nos objetos macroscópicos, é tão acentuada que se transforma em lei determinista.

A noção probabilística de entropia introduzida por Boltzmann permitiu que, em 1948, Claude Shannon (1916-2001) definisse entropia dentro do contexto da teoria da informação (uma teoria matemática-estatística que trata da transmissão de dados). ${ }^{7}$ Posteriormente, Léon Brillouin (1889-1969), em seu livro Science and Information Theory, ${ }^{8}$ verificou a equivalência entre os conceitos de entropia propostos por Clausius e Shannon, o que permitiu definir entropia em termos da informação estrutural do sistema.

Tradicionalmente, a termodinâmica clássica restringe o conceito entropia aos estados de equilíbrio e aos processos reversíveis. Porém, ao contrário da termodinâmica clássica, a mecânica dos meios contínuos (da qual a temporal termodinâmica dos processos homogêneos é uma radical simplificação porque, nesta última, é imposta a homogeneidade espacial de todas as propriedades intensivas) utiliza a entropia para relacionar as respostas térmicas e mecânicas do sistema em qualquer estado, pertencente a qualquer processo termodinâmico real.

Este trabalho tem por objetivo rediscutir a segunda lei da termodinâmica, no contexto da termodinâmica dos processos homogêneos, bem como os conceitos de temperatura e entropia, exclusivamente em termos das características intrínsecas do sistema. Dados estes conceitos, o estudo da desigualdade de Clausius e a definição e utilização das energias de Helmholtz e de Gibbs são consequências imediatas e extremamente úteis. Por outro lado, o conceito de entropia exige uma alusão ao teorema de Nernst sobre o calor.

\section{INFORMAÇÃO ESTRUTURAL FALTANTE E ENTROPIA}

É possível determinar várias propriedades de um sistema homogêneo, tais como sua pressão, $\mathrm{p}$, temperatura, T e quantidade de matéria, n. Mas, embora o conhecimento destes três valores seja suficiente para definir o estado de um sistema gasoso homogêneo formado por uma única substância quimicamente inerte, tal conhecimento nada informa sobre as posições e velocidades das partículas que constituem o sistema. Contendo este $\mathrm{N}=\mathrm{nN}_{\mathrm{A}}\left(\mathrm{N}_{\mathrm{A}}\right.$ é a constante de Avogadro) 
partículas, ter-se-ia que conhecer os valores de $6 \mathrm{~N}$ variáveis (para cada partícula, as três componentes cartesianas do seu vetor posição e as três do seu vetor velocidade) para descrever a configuração microscópica do sistema.

Portanto, enquanto que para conhecer o estado deste sistema, a cada instante, são suficientes os valores de três variáveis naquele momento, para conhecer o microestado deveriam ser conhecidos os valores de um número de variáveis da ordem de $10^{23}$. Como tal informação não é obtenível, a cada estado são associados todos os microestados com ele compatíveis, conforme propôs Boltzmann, atribuindo-se igual probabilidade de ocorrência a cada um deles.

Mas um processo homogêneo que transforme o estado representado por $\left(\mathrm{p}_{1}, \mathrm{~T}_{1}, \mathrm{n}_{1}\right)$ no estado representado por $\left(\mathrm{p}_{2}, \mathrm{~T}_{2}, \mathrm{n}_{2}\right)$ é uma sequência de infinitos estados que se modificam de forma contínua, porque as três variáveis envolvidas se alteram de modo contínuo. Isto indica que não se pode atribuir a cada estado um número inteiro de microestados, caso contrário ou todos os processos apenas envolveriam estados com igual quantidade de microestados, ou a menor diferença entre estados com quantidades diferentes de microestados seria igual a um microestado, ou seja, a diferença mínima em quantidade de microestados necessariamente introduziria uma descontinuidade na evolução dos estados, negando a afirmação de que se trata de uma sequência de infinitos estados que se modificam de forma contínua.

Por isto, define-se a densidade de microestados por estado, $\gamma$, em cada estado de um sistema, sendo $\gamma$ um adimensional cuja variação é contínua durante qualquer processo. Como nenhum estado pode corresponder a menos do que um microestado, ${ }^{9}$ tem-se $\gamma \geq 1$. Mas, para um sistema com um número de partículas da ordem de $10^{23}$ (este número tem 24 algarismos antes da vírgula), cujo estado apresente um valor de entropia da ordem de $1 \mathrm{~J} / \mathrm{K}$, a densidade de microestados é da ordem de $10^{\mathrm{A}}$, sendo $\mathrm{A}=10^{23}$ (este número tem $1+10^{23}$ algarismos antes da vírgula). Isto sublinha o absurdo que seria, para os costumeiros sistemas termodinâmicos, diferenciar $\gamma$ de $\gamma \pm 1$, ou seja, considerar que $\gamma$ não variasse de modo contínuo. Mostra, também, que sistemas macroscópicos correspondem a densidades colossais de microestados. De acordo com o teorema $\mathrm{H}$, tais densidades transformam tendências estatísticas em leis deterministas.

Seja adjetivada isolante uma fronteira impermeável à massa e à energia, quaisquer que sejam as condições do exterior ao sistema por ela delimitado e do próprio sistema assim isolado. Em qualquer sistema (não necessariamente isolado), defina como informação estrutural a qualquer informação sobre o sistema, desde que tal informação seja adicional em relação àquelas correspondentes a valores que necessariamente seriam constantes se o sistema fosse isolado. A informação estrutural faltante, $\psi$, é uma propriedade cujo aumento de valor determina a diminuição na quantidade de informação estrutural disponível sobre o sistema.

Para um sistema isolado, considera-se que $\psi$ apresente as seguintes características: $^{10,11}$ (i) o real $\psi$ deve ser uma função não negativa de $\gamma$, $\operatorname{logo} \psi(\gamma) \geq 0$ e, além disso, $\psi$ deve ser uma função contínua estritamente crescente de $\gamma$. Portanto, quanto maior a densidade de microestados, maior será a informação estrutural faltante para que seja conhecida toda a informação estrutural sobre o sistema. (ii) Caso, em algum instante $t$, um observador localizado no interior do sistema conhecesse tudo sobre este, ele conheceria o exato microestado em que se encontrasse o sistema naquele instante, portanto, ter-se-ia $\gamma=1$ e a informação estrutural faltante assumiria o valor zero, $\log o \psi(1)=0$. (iii) Se o sistema isolado fosse dividido em diversos sistemas isolados menores, a informação estrutural faltante do sistema original seria a soma das informações estruturais faltantes dos sistemas menores, enquanto que a densidade de microestados do sistema original seria o produto das densidades de microestados dos sistemas menores. Portanto, a informação estrutural faltante é aditiva, enquanto que a densidade de microestados é multiplicativa. Isto pode ser matematicamente representado por $\psi\left(\gamma_{1} \times\right.$ $\left.\gamma_{2} \times \ldots \times \gamma_{n}\right)=\psi\left(\gamma_{1}\right)+\psi\left(\gamma_{2}\right)+\ldots+\psi\left(\gamma_{n}\right)$.

Impondo estas três características a $\psi$, Shannon estabeleceu que:

$$
\psi=c \log _{a} \gamma
$$

onde $c$ é a unidade de medida da informação, determinada pela base $a$ dos logaritmos considerados. Por exemplo, quando logaritmos em base dois forem empregados $(a=2)$ ter-se-á $c=1$ bit, $\operatorname{logo}$, como $\log _{2}$ $\gamma=\ln \gamma / \ln 2$, encontrar-se-á $\psi=(\ln \gamma / \ln 2)$ bit. Portanto, se num sistema isolado a densidade de microestados do estado considerado for $\gamma$, serão necessários $(\ln \gamma / \ln 2)$ bit para armazenar a informação estrutural faltante daquele estado. Para qualquer base $a$, a Equação 1 pode ser escrita como:

$$
\psi=(c / \ln a) \ln \gamma
$$

Mas, quando $(c / \ln a)$ for igual à constante de Boltzmann $\mathrm{k}_{\mathrm{B}}$ $\left(1,3806488 \times 10^{-23} \mathrm{~J} / \mathrm{K}\right)$, a Equação 2 será a familiar expressão que relaciona a entropia com a densidade de microestados de um sistema isolado,

$$
\mathrm{S}=\mathrm{k}_{\mathrm{B}} \ln \gamma
$$

A razão entre as Equações 2 e 3 indica que a entropia é a informação estrutural faltante, porque $\mathrm{S} / \psi=\left(\mathrm{k}_{\mathrm{B}} \ln a\right) / c$. Por exemplo, para $a=2$ tem-se $\mathrm{S}=\mathrm{k}_{\mathrm{B}} \ln 2(\psi / b i t)$, ou

$$
\mathrm{S} /(\mathrm{J} / \mathrm{K})=9,569928 \times 10^{-24} \psi / \text { bit }
$$

As Equações 1, 2 e 3 são específicas para sistema isolado. Porém, a Equação 4 é válida para qualquer sistema, porque se trata, apenas, de uma transformação de unidades. Portanto, em qualquer sistema a entropia pode ser interpretada como a informação estrutural faltante do estado considerado.

A Equação 3, juntamente com o teorema $\mathrm{H}$ de Boltzmann, implica na entropia de um sistema isolado jamais diminuir quando o tempo avançar. Logo, o efeito conjunto da primeira e da segunda lei resulta em que, para sistema isolado, com o passar do tempo a energia interna seja redistribuída sem que a sua quantidade total seja alterada, enquanto que a entropia aumente até um valor máximo, quando então o sistema atinge o seu estado estável. Pode-se, também, considerar que o estado estável de um sistema isolado maximize a entropia porque as informações sobre as condições iniciais do sistema tendem a ser perdidas, exceto aquelas referentes às quantidades que, em tal sistema, são necessariamente conservadas. Consequentemente, a maximização da entropia de um sistema isolado é a maximização da nossa ignorância sobre o mesmo. ${ }^{12}$

Para um sistema fechado (fronteira impermeável à massa), mas não isolado, a proporcionalidade entre entropia e logaritmo da densidade de microestados não é mantida, porque valor de $\gamma$ é afetado pelo fato do conjunto de propriedades que não variam em um sistema isolado ser mudado para outro conjunto de propriedades invariantes, enquanto que a entropia continua proporcional à informação estrutural faltante. Porém, existirá outro tipo de informação faltante, embora não mais aquela que foi adjetivada estrutural, que satisfaça as condições listadas de (i) a (iii), ou seja, que satisfaça uma expressão análoga à Equação 1. Quando, em tal expressão, a constante de proporcionalidade for a constante de Boltzmann, será obtida uma igualdade análoga à Equação 3,

$$
\chi=\mathrm{k}_{\mathrm{B}} \ln \gamma,
$$

onde $\chi$ jamais poderá diminuir à medida que o tempo avançar e a 
maximização de $\chi$ corresponderá à maximização da nossa ignorância sobre o sistema.

Evidentemente, $\chi$ será a entropia $\mathrm{S}$ se o sistema for, além de fechado, também isolado (neste caso, a informação faltante será estrutural). Mas, para melhor entender a Equação 5, considere um sistema homogêneo, fechado por uma fronteira impermeável a toda energia salvo volumétrico (o trabalho envolvido quando o volume do sistema varia), contendo uma única substância gasosa quimicamente inerte. Note que qualquer estado bem determinado deste sistema pode ser definido tanto pelo conjunto de valores $<\mathrm{T}$, $\mathrm{p}$, $\mathrm{n}>$, quanto pelo conjunto $<\mathrm{T}, \mathrm{V}, \mathrm{n}>$. De acordo com a termodinâmica estatística, ${ }^{13}$ dado o conjunto $<\mathrm{T}, \mathrm{p}, \mathrm{n}>$ tem-se $\ln \gamma=-\frac{\mathrm{G}}{\mathrm{k}_{\mathrm{B}} \mathrm{T}}$ ao passo que, para o conjunto $<\mathrm{T}, \mathrm{V}, \mathrm{n}>$, tem-se $\ln \gamma=-\frac{\mathrm{A}}{\mathrm{k}_{\mathrm{B}} \mathrm{T}}$, onde $\mathrm{G}$ e A são, respectivamente, as energias de Gibbs e de Helmholtz. Como $\mathrm{G}=\mathrm{A}$ $+\mathrm{pV}$ e, para todo sistema gasoso, $\mathrm{pV} \neq 0$, para um mesmo estado do sistema tem-se, necessariamente, $\mathrm{G} \neq \mathrm{A}$.

Portanto, considerar um mesmo estado em termos do conjunto $<\mathrm{T}, \mathrm{p}, \mathrm{n}\rangle$, ou em termos do conjunto $<\mathrm{T}, \mathrm{V}, \mathrm{n}\rangle$, corresponde a alterar o valor da sua densidade de microestados. Isto ocorre porque os microestados correspondentes à descrição do estado, em termos dos parâmetros (fixos) $<\mathrm{T}, \mathrm{p}, \mathrm{n}>$, não são os microestados usados para descrever o estado em termos dos parâmetros (também fixos) $<\mathrm{T}, \mathrm{V}$, $\mathrm{n}\rangle$. Se este mesmo estado fosse descrito pelo conjunto $\langle\mathrm{U}, \mathrm{V}, \mathrm{n}\rangle$, ou seja, fosse descrito considerando que energia interna, volume e quantidade de matéria fossem os valores fixos, ter-se-ia $\ln \gamma=\frac{\mathrm{S}}{\mathrm{k}_{\mathrm{B}}}$.

Note que o adimensional $\ln \gamma$, multiplicado pela constante de Boltzmann, é, conforme o caso, a própria entropia ou qualquer uma das demais propriedades entrópicas (propriedades análogas à entropia, tais como $-\frac{G}{T} e-\frac{A}{T}$ ). Estas foram definidas sem fazer menção a qualquer adjetivo como "desordenado", "caótico", etc. Considerar um estado mais ordenado do que outro pode ter caráter subjetivo e a associação entre entropia e caos, sem que esta última palavra tenha sido previamente definida em claros termos físicos, é falha. Sistemas inicialmente em estados metaestáveis fornecem inúmeros exemplos onde o estado final estável é mais ordenado (conforme critérios usuais) do que o estado inicial, embora o processo em questão sempre exija o aumento de alguma propriedade entrópica e, em alguns casos, até mesmo da própria entropia. ${ }^{14}$

\section{DESIGUALDADE DE CLAUSIUS}

Para sistema fechado termicamente homogêneo tem-se que ${ }^{5}$

$$
\frac{\mathrm{dq}}{\mathrm{d} t} \leq \mathrm{T} \frac{\mathrm{dS}}{\mathrm{d} t},
$$

onde T é a temperatura absoluta homogênea no instante $t$ e subentende-se que $\frac{\mathrm{dq}}{\mathrm{d} t}$ seja positivo no caso de ocorrer absorção de potência térmica naquele momento e negativo quando acontecer emissão. A Equação 6 indica que, se em determinado instante um sistema estiver absorvendo calor de modo termicamente homogêneo, naquele momento a sua entropia aumentará em uma velocidade no mínimo igual à razão entre a potência térmica absorvida e a temperatura. Por outro lado, se em determinado instante a entropia do sistema estiver diminuindo de modo termicamente homogêneo, naquele momento calor será emitido e a razão entre o módulo da potência térmica emitida e a temperatura será no mínimo igual ao módulo da velocidade de diminuição da entropia.
A Equação 6 também introduz a ideia de dissipação, que é a velocidade de alteração, no instante $t$, de uma grandeza do sistema grafada $\delta$. A dissipação, definida por $\frac{\mathrm{d} \delta}{\mathrm{d} t} \equiv \mathrm{T} \frac{\mathrm{dS}}{\mathrm{d} t}-\frac{\mathrm{dq}}{\mathrm{d} t}$, é análoga ao conceito de atrito, todavia trata-se de um atrito interno ao sistema e não entre o sistema e suas vizinhanças. Ela sempre ocorrerá quando, no estado considerado do sistema, houver tendência de alteração nos movimentos internos do sistema, os quais justificam a existência da energia interna. ${ }^{15}$ Se a definição de dissipação for substituída na Equação 6, ter-se-á

$$
\frac{\mathrm{d} \delta}{\mathrm{d} t} \geq 0
$$

Portanto, em sistemas fechados termicamente homogêneos ou a grandeza $\delta$ permanece constante (processos não dissipativos), ou aumenta (processos dissipativos). Se for adicionalmente imposto que o sistema seja mantido isolado de suas vizinhanças, de acordo com as Equações 6 e 7 ter-se-á $\frac{\mathrm{dS}}{\mathrm{d} t} \geq 0$ e $\frac{\mathrm{d} \delta}{\mathrm{d} t} \geq 0$. Desta forma, verifica-se que a dissipação poderá ocorrer mesmo que o sistema não troque energia com suas vizinhanças, reforçando o conceito de que a dissipação é um fenômeno interno ao sistema.

Cabe, ainda, comentar que a fronteira de um sistema não é uma parede física, mas sim um conceito matemático, porque se trata de uma superfície bidimensional, pertencente ao sistema e que o delimita, separando-o do seu exterior. Por isto, um ponto exterior pode aproximar-se sempre mais da fronteira, mas jamais a alcançará, enquanto que um ponto do sistema jamais ultrapassará a fronteira. A homogeneidade térmica do sistema, portanto, exige que o exterior aplique à fronteira do sistema uma temperatura idêntica à temperatura homogênea do sistema, caso contrário os pontos da fronteira possuiriam duas temperaturas. Logo, a transmissão de calor entre o sistema e seu exterior, através da fronteira, ocorre na temperatura homogênea do sistema. Isto indica que a Equação 6 é análoga à desigualdade divulgada por Clausius em 1862. Ela é a segunda lei da termodinâmica dos processos homogêneos.

\section{TEOREMA DE NERNST SOBRE O CALOR}

Apesar da Equação 3 mostrar que a entropia do sistema isolado assume o valor zero quando $\gamma=1$, isto não necessariamente indica que existam, na natureza, sistemas cuja entropia seja nula, ou seja, sistemas com $\gamma=1$. Por exemplo, sabe-se que em estados vítreos, mesmo que a temperatura absoluta do sistema tenda a zero, a entropia não tende a este valor. Esta afirmação, contudo, não contradiz o teorema proposto por Walther Nernst (1864-1941), sobre o calor. Tal teorema afirma que a variação da entropia, para qualquer transformação física ou química efetuada com o sistema em temperatura homogênea e constante, tenderá ao valor zero quando tal temperatura se aproximar do zero absoluto, logo

$$
\lim _{\mathrm{T} \rightarrow 0}(\Delta \mathrm{S})=0 \text {. }
$$

Porém, não há dúvida de que o valor da entropia, para qualquer substância cuja temperatura homogênea tenda ao zero absoluto, pode ser negligenciado quando comparado aos valores de entropia obtidos para a mesma substância, a 298,15 K. Esta afirmação esclarece o enunciado feito por Planck (1858-1947) em 1910, o qual declarou que, à medida que a temperatura diminuir indefinidamente, a entropia de um sistema químico homogêneo tenderá a zero, ${ }^{16}$ portanto

$$
\lim _{\mathrm{T} \rightarrow 0} \mathrm{~S}=0 .
$$




\section{TEMPERATURA}

Suponha que o estado de um sistema homogêneo constituído por $J$ espécies químicas possa ser caracterizado pelos valores apresentados, no instante $t$, pela sua entropia $\mathrm{S}$, volume $\mathrm{V}$ e quantidades de matéria $\mathrm{n}_{\mathrm{j}}, \mathrm{j}=1, \ldots, J$. Como a energia interna é uma função do estado do sistema no instante $t$, tem-se então $\mathrm{U}=\mathrm{U}\left(\mathrm{S}, \mathrm{V}, \mathrm{n}_{1}, \ldots, \mathrm{n}_{J}\right)$. Para exemplificar o conceito de função de estado, considere que o estado de um sistema homogêno, contendo uma única substância gasosa quimicamente inerte, seja definido pelo conjunto de valores $\langle\mathrm{T}, \mathrm{V}, \mathrm{n}\rangle$. Supondo que o gás se comporte idealmente, tem-se $\mathrm{p}=\mathrm{p}(\mathrm{T}, \mathrm{V}, \mathrm{n})=\frac{\mathrm{nRT}}{\mathrm{V}}$, ou seja, a pressão é função das variáveis que definem o estado, logo é uma função de estado. Porém, como $\mathrm{T}=\mathrm{T}(t), \mathrm{V}=\mathrm{V}(t)$ e $\mathrm{n}=\mathrm{n}(t)$, evidentemente $\mathrm{p}=\mathrm{p}(\mathrm{T}, \mathrm{V}, \mathrm{n})$ implica em $\mathrm{p}=\mathrm{p}(t)$, embora $\mathrm{p}=\mathrm{p}(t)$ não implique em $\mathrm{p}=\mathrm{p}(\mathrm{T}, \mathrm{V}, \mathrm{n})$ (uma função de estado é função temporal, mas nem toda função temporal é função de estado).

Definindo $\Phi=<\mathrm{S}, \mathrm{V}, \mathrm{n}_{1}, \ldots, \mathrm{n}_{J}>$, pode-se escrever $\mathrm{U}=\mathrm{U}(\Phi)$, a qual é uma função diferenciável para todo conjunto $\Phi$. Considerando a energia interna, a entropia, o volume e a quantidade de matéria como propriedades primitivas, as demais propriedades termodinâmicas, no sistema homogêneo, podem ser definidas a partir delas. ${ }^{17}$ Por exemplo, se em um sistema homogêneo os valores de $V$ e $n_{1}, \ldots, n_{J}$ forem mantidos constantes, a temperatura homogênea do sistema em cada estado $\Phi$ será a tendência da razão entre a variação da energia interna e a variação de entropia naquele estado, $T=\frac{\partial U}{\partial S}(\Phi)$. Além disso, porque a energia interna é uma função estritamente crescente da entropia, tem-se

$$
\mathrm{T}=\frac{\partial \mathrm{U}}{\partial \mathrm{S}}(\Phi)>0
$$

No entanto, as primeiras noções científicas de temperatura foram propostas por Kelvin em 1848, sem fazer qualquer menção aos conceitos de entropia e energia interna. Elas emergiram da termometria e a primeira escala de temperatura proposta por Kelvin envolveu o conceito de temperatura empírica, $\theta$. Kelvin supôs que os valores de $\theta$ formassem uma escala adimensional de temperaturas que incluísse todo o eixo dos reais, ou seja, $-\infty<\theta<+\infty$. Para transformar esta escala adimensional em dimensional, pode-se escolher alguma propriedade termométrica, $\Xi$, cuja derivada em relação a $\theta$ seja sempre positiva, $\log 0 \frac{\partial \Xi}{\partial \theta}>0$. Então, a escala dimensional é construída associando-se, arbitrariamente, dois valores da escala adimensional aos valores de $\Xi$ correspondentes a dois estados de referência bem determinados. Escalas definidas desta forma são conhecidas como escalas empíricas.

Se uma nova escala adimensional for definida a partir de $\mathrm{T}=$ $\exp (\theta)$, esta nova escala, ao contrário da primeira, incluirá apenas os reais positivos, $\log _{0} 0<\mathrm{T}<+\infty$. Pode-se relacionar o adimensional $\mathrm{T}=1$ à unidade Kelvin, a qual é, por definição, $\frac{1}{273,16}$ do valor da temperatura da água no seu ponto tríplice. Todavia, o adimensional 1 poderia ser associado a qualquer sistema em estado físico bem determinado. Escalas definidas desta maneira são denominadas escalas absolutas, ou termodinâmicas, ou de gás perfeito.

Elas são absolutas porque apresentam um valor finito como limite inferior e, por isto, ao invés da relação entre dois estados basta um único estado de referência para torná-las dimensionais. São chamadas termodinâmicas porque Kelvin mostrou que elas são uma consequência lógica do trabalho de Carnot, sobre máquinas térmicas. Adicionalmente, são consideradas de gás perfeito porque são coerentes com a extrapolação do comportamento dos gases rarefeitos para temperaturas tão baixas que, qualquer que seja a pressão, nenhuma substância real, em equilíbrio termodinâmico, permanece gasosa em tais temperaturas.

Como qualquer estado representável por $\Phi$ também pode ser caracterizado pelo conjunto de valores $\left.<\mathrm{T}, \mathrm{V}, \mathrm{n}_{1}, \ldots, \mathrm{n}_{J}\right\rangle$, a função $\mathrm{T}=\frac{\partial \mathrm{U}}{\partial \mathrm{S}}(\Phi)$ apresenta inversa. Isto exige que, se os valores de $\mathrm{V}$ e $\mathrm{n}_{1}, \ldots, \mathrm{n}_{J}$ forem mantidos constantes, a variação da entropia com a temperatura (ou vice-versa) seja ou sempre positiva, ou sempre negativa. De fato, $\frac{\partial \mathrm{T}}{\partial \mathrm{S}}(\Phi)>0$, o que indica que, quando $\mathrm{S}$ diminuir, T também diminuirá e vice-versa. Portanto, quando uma tender para a sua cota inferior máxima, o mesmo deverá acontecer com a outra. Logo, independentemente de qual seja o valor mínimo para o qual a entropia efetivamente tenda, como $\frac{\partial \mathrm{T}}{\partial \mathrm{S}}(\Phi)>0$ a inatingibilidade do zero absoluto de temperatura implica na inatingibilidade do mínimo de entropia e vice-versa. Como o mínimo de entropia é igual ou superior a zero, a inatingibilidade do zero absoluto implica na impossibilidade da entropia se anular, ou seja, na impossibilidade de obter total conhecimento sobre o estado de um sistema homogêneo isolado.

\section{ENERGIAS DE HELMHOLTZ E DE GIBBS}

Seja um sistema fechado termicamente homogêneo. Pelo emprego da expressão da primeira lei da termodinâmica em termos de derivadas temporais, $\frac{\mathrm{dU}}{\mathrm{d} t}=\frac{\mathrm{dq}}{\mathrm{d} t}+\frac{\mathrm{dw}}{\mathrm{d} t},{ }^{17}$ a Equação 6 pode ser reescrita como

$$
\frac{\mathrm{dU}}{\mathrm{d} t}-\mathrm{T} \frac{\mathrm{dS}}{\mathrm{d} t} \leq \frac{\mathrm{dw}}{\mathrm{d} t}
$$

que é a expressão matemática da segunda lei para o mencionado sistema, agora em termos de potência atérmica. Sendo a energia de Helmholtz de um sistema termicamente homogêneo definida por $\mathrm{A}=\mathrm{U}-\mathrm{TS}$, ter-se-á $\frac{\mathrm{dA}}{\mathrm{d} t}=\frac{\mathrm{dU}}{\mathrm{d} t}-\mathrm{T} \frac{\mathrm{dS}}{\mathrm{d} t}$ se e somente se o estado do sistema pertencer a um processo isotérmico (processo termicamente homogêneo em temperatura constante, $\log \frac{\mathrm{dT}}{\mathrm{d} t}=0$ ). Portanto, em todo estado pertencente a um processo isotérmico a Equação 11 pode ser escrita como

$$
\frac{\mathrm{dA}}{\mathrm{d} t} \leq \frac{\mathrm{dw}}{\mathrm{d} t}
$$

o que mostra ser a velocidade de aumento da energia de Helmholtz, no máximo, igual à potência atérmica absorvida, enquanto que o módulo da velocidade de diminuição da mesma energia é, no mínimo, igual ao módulo da potência atérmica emitida.

A potência atérmica pode ser considerada a soma de duas parcelas, a saber, uma parcela volumétrica, $\frac{\mathrm{dw}_{\mathrm{V}}}{\mathrm{d} t}$ e outra não volumétrica, $\frac{\mathrm{dw}_{\mathrm{NV}}}{\mathrm{d} t}$. Em um sistema fechado baricamente homogêneo $\frac{\mathrm{dw}_{\mathrm{V}}}{\mathrm{d} t}=-\mathrm{p} \frac{\mathrm{dV}}{\mathrm{d} t} \log 0$, neste sistema, tem-se $\frac{\mathrm{dw}}{\mathrm{d} t}=\frac{\mathrm{dw}_{\mathrm{NV}}}{\mathrm{d} t}-\mathrm{p} \frac{\mathrm{dV}}{\mathrm{d} t}$, que substituído na Equação 11 produz, para um sistema fechado termobaricamente homogêneo, a expressão

$$
\frac{\mathrm{dU}}{\mathrm{d} t}+\mathrm{p} \frac{\mathrm{dV}}{\mathrm{d} t}-\mathrm{T} \frac{\mathrm{dS}}{\mathrm{d} t} \leq \frac{\mathrm{dw}_{\mathrm{NV}}}{\mathrm{d} t}
$$


gêneo definida por $\mathrm{G}=\mathrm{U}+\mathrm{pV}$ - TS, ter-se-á $\frac{\mathrm{dG}}{\mathrm{d} t}=\frac{\mathrm{dU}}{\mathrm{d} t}+\mathrm{p} \frac{\mathrm{dV}}{\mathrm{d} t}-\mathrm{T} \frac{\mathrm{dS}}{\mathrm{d} t}$ se e somente se o estado do sistema pertencer a um processo isotermobárico (processo termobaricamente homogêneo em temperatura e pressão constante, $\left.\log 0 \frac{\mathrm{dT}}{\mathrm{d} t}=0 \mathrm{e} \frac{\mathrm{dp}}{\mathrm{d} t}=0\right)$.

Portanto, em todo estado pertencente a um processo isotermobárico a Equação 13 pode ser escrita como

$$
\frac{\mathrm{dG}}{\mathrm{d} t} \leq \frac{\mathrm{dw}_{\mathrm{NV}}}{\mathrm{d} t}
$$

o que mostra ser a velocidade de aumento da energia de Gibbs, no máximo, igual à potência atérmica não volumétrica absorvida, enquanto que o módulo da velocidade de diminuição da mesma energia é, no mínimo, igual ao módulo da potência atérmica não volumétrica emitida. Os processos químicos são, muitas vezes, considerados aproximadamente isotermobáricos, em sistemas fechados. Além disso, geralmente não se considera que potências atérmicas não volumétricas (elétricas, magnéticas, gravitacionais etc.) sejam trocadas entre o sistema e seu exterior. Por isto, para os processos químicos usuais a segunda lei da termodinâmica pode ser representada por $\frac{\mathrm{dG}}{\mathrm{d} t} \leq 0$, ou seja, a energia de Gibbs diminui a cada instante durante o processo.

Cabe comentar que, impondo-se isentropia (entropia constante durante o processo) a Equação 6 mostra que a potência térmica não pode ser positiva. A partir disto e aplicando-se imposições adicionais, desigualdades equivalentes às representadas nas Equações $12 \mathrm{e} 14$ podem ser facilmente deduzidas para a energia interna e a entalpia. Entretanto, condições como homogeneidade térmica e bárica, isotermia e isobaria são experimentalmente controláveis, enquanto que isentropia é um conceito teórico, não controlável por medição direta. Por isto, considera-se que a Equação 6 indique qual é a velocidade mínima de alteração de uma propriedade do sistema, dada uma controlável potência térmica transferida através de sua fronteira, ao invés de qual é a potência térmica máxima correspondente a uma não diretamente controlável velocidade de alteração da informação estrutural faltante no sistema.

\section{CONCLUSÃO}

A Equação 6 mostra que, num sistema termicamente homogêneo fechado por fronteira adiabática (impermeável ao calor), a entropia não pode diminuir, exatamente como ocorre em sistema isolado, onde não se exige homogeneidade térmica mas impõe-se fronteira isolante. Se, além de homogênea, a temperatura for um parâmetro (for fixa) e a fronteira do sistema fechado, ao invés de ser impermeável ao calor, for impermeável a qualquer tipo de trabalho, a Equação 12 indicará que a energia de Helmholtz não poderá aumentar. Por outro lado, se a temperatura e a pressão forem homogêneas e paramétricas e a fronteira do sistema fechado for impermeável apenas a trabalho do tipo não volumétrico, a Equação 14 garantirá que a energia de Gibbs não poderá aumentar.

Logo, estas condições do sistema e de sua fronteira são tais que o tipo de informação faltante, respectivamente representado por $\mathrm{S}$, $-\frac{\mathrm{A}}{\mathrm{T}} \mathrm{e}-\frac{\mathrm{G}}{\mathrm{T}}$, com valores dados em $\mathrm{J} / \mathrm{K}$, seja igual ao produto da constante de Boltzmann pelo logaritmo da correspondente densidade de microestados do estado do sistema, densidade esta que não pode diminuir. De fato, esta interpretação é coerente com a discussão colocada após a Equação 5, onde as conclusões obtidas por termodinâmica estatística não consideram a possibilidade de transmissão de potência atérmica não volumétrica, através da fronteira do sistema.

Mas suponha que as restrições de fronteira sejam retiradas. Por exemplo, no caso da Equação 6 considere que a fronteira não seja adiabática. Esta equação, então, mostra que a absorção de potência térmica exige que a informação faltante (no caso, estrutural) aumente em uma velocidade não inferior à razão entre a velocidade de absorção de calor pelo sistema e a temperatura homogênea do mesmo, no estado considerado. Isto é incompatível com a igualdade entre a informação estrutural faltante e o produto da constante de Boltzmann pelo logaritmo da correspondente densidade de microestados do estado do sistema (Equação 3), porque não pode ser imposto que a velocidade de aumento da densidade de microestados apresente um valor mínimo positivo, já que a segunda lei apenas afirma que não pode ocorrer diminuição. Por outro lado, a emissão de potência térmica permitiria que diminuísse a densidade de microestados, o que também seria um absurdo, de acordo com a segunda lei.

Portanto, o logaritmo da densidade de microestados será proporcional à informação estrutural faltante, na Equação 6, só quando a fronteira for adiabática. Ao se retirar esta restrição os microestados passam a ser de outro tipo, em consequência a densidade deles se altera e a proporcionalidade desaparece. O logaritmo desta nova densidade manterá proporcionalidade com algum outro tipo de informação faltante, diferente daquele correspondente à fronteira adiabática, que é a informação estrutural faltante. Mas, sendo ou não adiabática a fronteira, a Equação 6 impõe uma restrição à velocidade de alteração da informação estrutural faltante. Conclusões análogas podem ser obtidas para as Equações 12 e 14. Note que, de acordo com a termodinâmica dos processos homogêneos, a segunda lei é sempre interpretada como uma restrição à velocidade de resposta do sistema a estímulos externos.

\section{AGRADECIMENTOS}

M. C. Reis agradece ao CNPq por Bolsa de Doutorado concedida.

\section{REFERÊNCIAS}

1. Carnot, N. L. S.; Refléxions sur la Puissance Motrice du Feu et sur les Machines Propres à Développer Cette Puissance, Gauthier-Villars: Paris, 1878.

2. Šilhavý, M.; The Mechanics and Thermodynamics of Continuous Media, Springer: Berlin, 1997.

3. Kestin, K.; The Second Law of Thermodynamics, Dowden, Hutchinson \& Ross: Stroudsburg, 1976.

4. Serrin, J.; Arch. Ration. Mech. Anal. 1979, 70, 355.

5 Truesdell, C. A.; Rational Thermodynamics, $2^{\text {nd }}$ ed., Springer: New York, 1984.

6. Truesdell, C. A.; The Tragicomical History of Thermodynamics, 18221854, Springer: Heidelberg, 1980.

7. Shannon, C. E.; AT\&T Tech. J. 1948, 27, 379.

8. Brillouin, L.; Science and Information Theory, $2^{\text {nd }}$ ed., Dover: Mineola, 2004.

9. Fermi, E.; Thermodynamics, Dover: New York, 1956.

10. http://chemkeys.com/br/2005/11/18/entropia-e-energias-de-helmholtze-de-gibbs, acessada em Dezembro 2011.

11. Ben-Naim, A.; A Farewell to Entropy: Statistical Thermodynamics Based on Information, World Scientific: Toh Tuck Link, 2008.

12. Reis, M. C.; Bassi, A. B. M. S. Em Thermodynamics; Tadashi, M., ed.; Intech: Rijeka, 2011, cap. 5.

13. Tolman, R. C.; The Principles of Statistical Mechanics, Dover: New York, 1979.

14. Debenedetti, P. G.; Metastable Liquids: Concepts and Principles, Princeton University Press: Princeton, 1996.

15. http://chemkeys.com/br/2006/04/17/as-duas-expressoes-fundamentaisda-termodinamica, acessada em Novembro 2011

16. Planck, M.; Treatise on Thermodynamics, $3^{\text {rd }}$ ed., Dover: New York, 1945.

17. Moreira, N. H.; Bassi, A. B. M. S.; Quim. Nova 2001, 24, 563; Nery, A. R. L.; Bassi, A. B. M. S.; Quim. Nova 2009, 32, 522. 